\title{
Understanding Interrelationships between Body Dysmorphic Disorder with Anorexia Nervosa and Anxiety
}

\author{
Rangaprakash Deshpande ${ }^{1}$, Amoolya D. Vayalapalli ${ }^{2, *}$ \\ ${ }^{1}$ Department of Psychiatry and Biobehavioral Sciences, University of California, United States \\ ${ }^{2}$ Northview High School, United States
}

Copyright $\mathrm{O} 2017$ by authors, all rights reserved. Authors agree that this article remains permanently open access under the terms of the Creative Commons Attribution License 4.0 International License

\begin{abstract}
People have become more aware of body dysmorphic disorder and are actively seeking treatments to cure this mental disorder. Individuals affected with body dysmorphic disorder generally experience high comorbidity rates with other debilitating mental disorders, namely anorexia nervosa and anxiety. By collating information about the effects of body dysmorphic disorder with other mental disorders by using functional MRI, the neuroanatomy of body dysmorphic disorder can be dismantled to understand the interplay of dysfunctions between various mental disorders. The improved understanding could have clinical implications related to better treatments because of a more thorough comprehension of the pathophysiology of body dysmorphic disorder. Furthermore, research can expand into the analyzing of various mental activities in a resting state to further develop a more holistic interpretation of the effect of mental disorders on the brain.
\end{abstract}

Keywords Body Dysmorphic Disorder, Anorexia Nervosa, Anxiety, Functional MRI, Visual Processing

\section{Introduction}

Body Dysmorphic Disorder (BDD) is a psychiatric disorder with considerable prevalence in the general society of about $2 \%$ [21, 25]. This psychiatric disorder is characterized by obsessive preoccupations with one's own physical appearance, which can create significant impairment and impede one's life. With more than $13 \%$ of the psychiatric population $[10,18,26]$ affected by BDD, the cause of the disorder is still idiopathic, or unknown. The symptoms of BDD have considerable overlap with the symptoms exhibited by patients with many other mental disorders such as anorexia nervosa and generalized anxiety; the subtle differences between the different pathophysiological diseases are created by relatively small differences in the brain's processing of the exterior world. Impairments in the visual processing system create morphological changes that stem aberrant psychological behaviors.

Although the etiology of BDD is less understood, researchers agree that $\mathrm{BDD}$ affected patients perceive their faces as aversive because of aberrant visual processing [6]. Aberrant visual processing has been studied through a variety of brain imaging techniques, while each discovery brings us closer to finding more effective treatments for individuals with BDD. The associations between BDD and other mental disorders provide a more in depth perspective on the different morphological expressions and explain the high comorbidity rates between the various mental illnesses. The information synthesized in this review further promotes research in the abnormal visual processing of patients affected with BDD to help alleviate the symptoms of BDD and other comorbid mental disorders.

\section{Anorexia Nervosa and Body Dysmorphic Disorder}

Though BDD shares similar symptomatic characteristics with AN, they are morphologically different possibly due to discrepancies within the visual processing stream though results are inconsistent [14]. In BDD, Low Spatial Frequency (LSF) connectivity within the occipito-temporal faceprocessing is compromised whereas with $\mathrm{AN}$, there are abnormalities, but not to the extremes as suggested in BDD [14]. These distinctions serve to justify that AN affected patients more often scrutinize their body than their face, whereas for patients with BDD, the affected examine their faces $[16,19]$. By focusing on their bodies, AN affected patients feel the compulsive need to restrict their diet or abstain from eating. The predominant phenotypic difference between BDD and AN- imbalanced perceptions in detailed vs. holistic processing- arise due to the deficit in the construction of the low- resolution holistic template of the visual field, which are more pronounced in BDD [14]. Both BDD and AN share ritualistic behaviors such as checking their bodies in the mirror and measuring their bodies, and 
thus provoking a heightened self-consciousness [11]. The interesting fact that arises due to this research is that mental disorders may share symptoms that may cause similar misperceptions, but their smaller differences cause considerable distinctions between the phenomenology of the disorders. If treated wrongly, the body image misconceptions persist and can lead to harrowing lives. But psychiatric literature does little to distinguish the two because of a lack of quality research to implore upon these topics further [11].

These studies are crucial because they provide evidence that these mental disorders arise from different parts of the brain but might be interdependent which leads to the high comorbidity between BDD and eating disorders- more than $32 \%$ of patients report BDD with an eating disorder [23]. Though there might be some interconnectivity, the differences when magnified signal the diagnosing of divergent mental diseases. Having recognized that BDD is characterized by misperceived visual perception especially of the face, experimenting with only face stimuli is more salient to BDD than it is to AN [28]. The studies could have enhanced their experimentation by testing the BDD, AN, and healthy control (HC) groups with a variety of tests which include not just processing of face images, but total body images as well. By doing so, they would have been able to identify the precise discrepancies for their respective mental disorders and understand the various stimuli that can cause the irregular visual processing stream to become activated. Furthermore, the studies could have perhaps researched upon the influences of nature and nurture to develop a more thorough study. The role of gender carries a significant weight, especially because females are more willing to admit to their mental disorders whereas men seem to hide behind the social stigma that shadow these diseases- those who feel embarrassed or ashamed are more reluctant to share their symptoms [15]. By taking a family and childhood history, the studies could have provided a more holistic vision into what causes the two diseases and understand the stimulus which catalyzes the mental illness [15]. Cognitive behavioral treatment for those with $\mathrm{AN}$ has been proven to create functional brain activity changes with significant differences in the amygdala- these changes helped normalize the activation of the amygdala when shown fearful and happy faces. These negative suppression treatments can also be applied to those with BDD, and by applying these therapeutic means to change the course in which neurons are connected, we can see an increase in brain activation when patients viewed their own bodies afterwards [15]. The neural patterns could be studied more in depth, and we can try to assess a clear connection between physical discrepancies within the brain to further assess the comorbidity between AN and BDD [15]. Additionally, by understanding how genetics and life experiences interact, we could focus more on prophylactic care than trying to undertake a precarious situation of damage control. Contributing to further research about mental disorders, these studies acknowledge the physical but microscopic issues that cause mental disorders, persistent and prevalent.

\section{Anxiety and Body Dysmorphic Disorder}

BDD has a high comorbidity rate with many mental disorders, especially with anxiety $[2,28]$, the distress related to an imminent foreboding outcome. The amygdala integrates its surroundings to process fear which leads to the fight or flight response; constant over- stimulation of the amygdala is related to anxiety [20, 24]. Patients with BDD are often times mistaken with having social anxiety disorder (a comorbidity rate of $11 \%$ ) because they appear distant and shy in social settings [3]. Moreover, research has shown that connections with the ventral visual stream (VVS) and the amygdala create enhanced emotional and visual stimuli creating a junction for the mediatory paths between BDD and anxiety [2]. Both anxiety and BDD illustrate a brain morphology that correlates with symptom severity; they have a distinct relationship when processing visual and emotional stimuli because the symptom anxiety associated with BDD is due to the aberrant VVS for own face and familiar face viewing [2]. Similar with AN, BDD and anxiety share certain ritualistic behaviors and the stress from these repetitive behaviors further compounds the intrusive behaviors and increase the negative self-destructive thought process [15]. But the problem persists of determining whether BDD or anxiety are a comorbid feature and if a patient would benefit from receiving therapy solely for the former or the later to cure both the coexisting diseases.

The study highlights the existence of the relationship between BDD and anxiety, illustrating its peculiar association. Sharing similar pathways, the comorbidity rate is high, but anxiety may be a dimension of BDD according to the study. The study's comparison of own- face and familiarface testing proves that there was not a significant difference, hence anxiety is more associated with the limbic system rather than to symptom provocation. As anxiety increases, the patient perceives himself/ herself as more disfigured and ugly impairing activities of daily living (ADL). Therefore therapeutic research to calm anxiety could benefit patients affected with BDD to reduce symptoms that cause BDD. By doing so, the once continuous cycle of anxiety caused by an over-stimulated amygdala will be stopped. But to even imply the benefits of such therapeutic aspects to cure BDD with a comorbidity of anxiety requires understanding etiology, which still alludes us [22]. It is generally believed to spark during early adolescence, when many teenagers are more critical about their appearance. Even yet, the morphology of this disease can arise from biological, psychological, and/ or environmental stimuli [22].

The study, however, deals with strong correlational events between Hamilton Depression Rating Scale (HDRS) and Hamilton Anxiety Rating Scale (HARS) which do not imply causation $[12,13]$. Also, the study could have researched a 
more specific part of the ventral visual stream (VVS) rather than the holistic view of the effects of anxiety on the VVS to eliminate any errors in inferences specific to face processing. Though the conducted study could have made a few adjustments, the research is invaluable because it highlights that anxiety can exacerbate the effects of BDD rather than reinforcing the misconstrued conception that BDD causes anxiety or vice versa [22]. In this manner, the primary disease is treated with symptomatic alleviation of comorbid disorders [22].

\section{Visual Processing (Object and Figure)}

Individuals affected with BDD focus on the smallest of details that affect their body- from their asymmetrical nose to tiny discolored dots on their foreheads. A study conducted found that those affected with BDD maintain an underactive configural and holistic elements of their visual processing while only remembering specific visual details [4]. The same study also concluded that patients may have had difficulty from switching from one self- related task to a stimulus independent task. Patients with BDD have often displayed a hyperactive local visual system, rather than their $\mathrm{HC}$ counterparts who employ a more global visual stream [1]. The behavior engaged by those affected with BDD can be associated with more local processing brain structures, but the question awaits whether or not BDD is a result of a localized visual processing stream or the vice versa [1].

The abnormalities encountered in the visual processing stream of those with BDD can be created by a variety of adverse effects, but individuals with BDD feel as if their appearance is contorted, triggering emotional arousal and/ or stimulating compulsive behaviors [27]. The comparison between own- face and other- face stimuli was crucial towards compiling data that visual abnormalities in lower-level processing persist for those with symptoms of BDD, whether it may be the result of top down attentional modulation or intrinsic aberrant visual processing system [17]. Furthermore, similar research conducted on visual processing looked at own- face processing and determined that the distorted perceptions are the result of impaired capacity to perceive low resolution pathways of the image because of a relative deficit of dorsal- stream magnocellular pathways [6]. Additionally, those with BDD seem to be less susceptible to any inversion schemes- stimuli are rotated 180 degrees from their original orientation. Those in the HC group, because of their global processing remain unfamiliar and are more sensitive to the newly configured stimulus, whereas their counterparts measure the stimulus through their highly trained local visual processing streams $[1,5]$.

Because the temporal resolution of fMRI testing results in an imprecise visual acuity to distinguish between stimuli encountered from a concentrated effort to find a goal or aberrant visual processing, it prohibited certainty within both cases. Though the studies conducted could have found ways to make their analysis of their experimentation more reliable, they were important in discerning that BDD affected individuals have an irregular visual system, both with higherand lower- processing and global and local processing. These differences suggest that people affected with BDD have a unique perspective of viewing their surroundings that creates a self-destructive lifestyle. The pattern of compulsive obsessive thoughts can be triggered by own- and other- face stimuli as a result of an inherent thought process that criticizes one's body for their perceived malformations. Because BDD affected individuals perceive everything and their triggers may arise from minute details, treating their pathophysiological processing may be difficult. But preventing by reducing predispositional or environmental stimuli or treating with cognitive behavioral therapy, these self-consuming and fatally destructive thoughts can provide reprieve to individuals who encounter these repetitive thoughts [5].

\section{Conclusions}

By becoming more cognizant of the neuroanatomy of the brain and how its various morphological components are becoming astray, the pathophysiology of BDD may finally be discovered. The tentative model of the dysfunction that causes BDD is a result of analyzing the symptoms, but these may belie the true origins of BDD. By stimulating individuals with more rigorous testing to decipher abnormalities in not just visual processing, but also other information processing systems, further research could facilitate improved treatments and therapy for this debilitating disorder. Furthermore, analyzing different mental disorders and their comorbidity rates could allow us to apprehend the different regional brain volumes that are directly affected and could help explore the interconnections that cause the disarray. As the relationships between different mental disorders progresses, the ability to treat individuals effectively increases whether it is through somatic and/ or psychotherapeutic means.

\section{Disclosures}

The author reports no competing interests.

\section{REFERENCES}

[1] Beilharz F, Atkins K, Duncum A, Mundy M. Altering visual perception abnormalities: a marker for body image concern. PloS ONE. 2016; 11(3): e0151933.

[2] Bohon, C., Hembacher, E., Moller H., Moody, T., Feusner J., Nonlinear relationships between anxiety and visual processing of own and others' faces in body dysmorphic disorder. Elsevier. Psychiatry Research: Neuroimaging. 2012; 204: 132139. 
[3] Fang A, Hofmann S. Relationship between social anxiety disorder and body dysmorphic disorder. Clinical psychology review. 2010; 30(8):1040-1048.

[4] Feusner, J., Hembacher, E., Moller, H., \& Moody, T. Abnormalities of object visual processing in body dysmorphic disorder. Cambridge University Press. Psychological Medicine. 2011; 41: 2385- 2397.

[5] Feusner J, Moller H, Altstein L, Sugar C, Bookheimer S, Yoon $\mathrm{J}$, Hembacher E. Inverted face processing in body dysmorphic disorder. Journal of psychiatric research. 2010; 44(15):1088-1094.

[6] Feusner, J., Moody, T., Hembacher, E., Townsend, J., McKinley, M., Moller, H., Bookheimer, S. Abnormalities of visual processing and frontostriatal systems in body dysmorphic disorder. American Medical Association. Arch Gen Psychiatry. 2010; 67 (2): 197-205.

[7] Feusner, J., Townsend, M., Bystritsky, A., Bookheimer, S. Visual information processing of faces in body dysmorphic disorder. American Medical Association. Arch Gen Psychiatry. 2007; 64 (12): 1417- 1426.

[8] Feusner, J., Townsend, M., Bystritsky, A., McKinley, M., Moller, H., \& Bookheimer, S. Regional brain volumes and symptom severity in body dysmorphic disorder. Elsevier. Psychiatry Research: Neuroimaging. 2009; 172: 161- 167.

[9] Feusner, J., Yaryura- Tobias, J., \& Saxena, S. The pathophysiology of body dysmorphic disorder. Elsevier. Body Image 5. 2008; 3-12.

[10] Grant, J., Kim, S., \& Crow, S. Prevalence and clinical features of body dysmorphic disorder in adolescent and adult psychiatric inpatients. Journal of Clinical Psychiatry. 2001; 62: 517- 522.

[11] Grant J, Phillips K. Is anorexia nervosa a subtype of body dysmorphic disorder? Probably not, but read on.... Harvard review of psychiatry. 2004; 12(2):123-126.

[12] Hamilton M. A rating scale for depression. Journal of Neurology, Neurosurgery, \& Psychiatry. 1960; 23:56-62.

[13] Hamilton M. Diagnosis and rating of anxiety. British Journal of Psychiatry. 1969; 3:76-79.

[14] Li, W., Lai, T., Bohon, C., Loo, S., McCurdy, D., Strober, M., Bookheimer, S., \& Feusner, J. Anorexia Nervosa and Body Dysmorphic Disorder are associated with abnormalities in processing visual information. Cambridge University Press. Psychological Medicine. 2015; 45: 2111-2122.

[15] Madsen S, Bohon C, Feusner J. Visual processing in anorexia nervosa and body dysmorphic disorder: similarities, differences, and future research directions. Journal of Psychiatric research. 2013; 47(10):1483-1491.

[16] Moody, T., Sasaki, M., Bohon, C., Strober, M., Bookheimer, S., Sheen, C., \& Feusner, J. Functional connectivity for face processing in individuals with body dysmorphic disorder and anorexia nervosa. Cambridge University Press. Psychological Medicine. 2015; 45: 3491-3503.

[17] Morris JS, Friston KJ, Buchel C, Frith CD, Young AW, Calder AJ, Dolan RJ. A neuromodulatory role for the human amygdala in processing emotional facial expressions. Brain. 1998; 121:47-57.

[18] Phillips KA, Nierenberg AA, Brendel G, Fava M. Prevalence and clinical features of body dysmorphic disorder in atypical major depression. Journal of Nervous and Mental Disorders. 1996; 184:125-129.

[19] Phillips KA. The Broken Mirror. Oxford University Press; New York: 2005.

[20] Ressler, K. Amygdala Activity, fear, and anxiety: modulation by stress. Biological Psychiatry. 2010; 67(12): 1117- 1119.

[21] Rief, W., Buhlmann, U., Wilhelm, S., Borkenhagen, A., \& Brahler, E. The prevalence of body dysmorphic disorder: a population based survey. Psychological Medicine. 2006; 87785.

[22] Ross J, Gowers S. Body dysmorphic disorder. Advances in psychiatric treatment. 2011;17:142-49.

[23] Ruffolo J, Phillips K, Menard W, Fay C, Weisberg R. Comorbidity of body dysmorphic disorder and eating disorders: severity of psychopathology and body image disturbance. International Journal of Eating Disorders. 2006; 39: 11-9.

[24] Stein MB, Goldin PR, Sareen J, Zorrilla LT, Brown GG. Increased amygdala activation to angry and contemptuous faces in generalized social phobia. Archives of General Psychiatry. 2002; 59:1027-1034.

[25] Veale, D., Gledhill, L., Christodoulou, P., \& Hodsoll, J. (2016). Body Dysmorphic Disorder in different settings: A systematic review and estimated prevalence. Body Image, 18, 168- 86.

[26] Wilhelm S, Otto MW, Zucker BG, Pollack MH. Prevalence of body dysmorphic disorder in patients with anxiety disorders. Journal of Anxiety Disorders. 1997; 11:499-502.

[27] Yaryura-Tobias J, Neziroglu F, Chang R, Lee S, Pinto A, Donohue L. Computerized perceptual analysis of patients with body dysmorphic disorder. CNS Spectrums. 2002; 7:444-446.

[28] Zimmerman M, Mattia JI. Body dysmorphic disorder in psychiatric outpatients: recognition, prevalence, comorbidity, demographic, and clinical correlates. Compr Psychiatry. 1998; 39:265-270.

[29] Online Available:

https://mghocd.org/bdd-and-anorexia-nervosa-what $\% \mathrm{E} 2 \% 80$ $\% 99$ s-the-big-difference-is-there-a-difference/: Reuman L, BDD and Anorexia NErvosa: What's the Big Difference? Is there a Difference?, Online available from https://mghocd.org/ 